\title{
Thermal relaxation of field-induced irreversible ferromagnetic phase in Pr-doped manganites
}

\author{
Joonghoe Dho and N. H. Hur* \\ Center for CMR materials, Korea Research Institute of Standards and Science, Yuseong P.O.Box \\ 102, Daejon 305-600, Korea
}

(July 20, 2021)

\begin{abstract}
We have investigated the magnetic and transport properties of $\operatorname{Pr}_{0.65} \mathrm{Ca}_{0.35} \mathrm{MnO}_{3}$ and $\operatorname{Pr}_{0.82} \mathrm{Na}_{0.18} \mathrm{MnO}_{3}$ with almost same doping level under various magnetic fields. Upon applying a field of $4 \mathrm{~T}$, both compounds undergo a metamagnetic transition from antiferromagnetic (AFM) to ferromagnetic (FM) in the low temperature region. The field-induced FM state is very stable for at least $10^{5} \mathrm{~s}$ even after removing the field. This irreversible FM behavior is different from the spontaneous FM ordering as well as the FM state in a reversible metamagnetic transition. We discuss this unusual FM phenomenon found in the Pr-doped manganites with the AFM ground state in terms of the reorientation of Mn spins pinned by localized Pr moments. PACS number : 75.30.Vn, 75.30.Kz, 75.60.Nt
\end{abstract}

Typeset using REVTEX 


\section{INTRODUCTION}

Over the last few years, a large amount of research has been undertaken towards understanding the physical properties of doped perovskite manganites, mainly because they exhibit a novel magneto transport property known as colossal magneto resistance (CMR) [1-3]. Among them, Pr-doped manganites $\mathrm{Pr}_{1-x} \mathrm{~A}_{x} \mathrm{MnO}_{3}(\mathrm{~A}=\mathrm{Ca}$, Sr, Na) have been the subjects of several studies due to their diverse physical and structural properties with doping concentration at different temperatures [4-11]. In the case of a $30 \%$ Ca doped sample, it undergoes a charge ordering $(\mathrm{CO})$ transition near $220 \mathrm{~K}\left(T_{C O}\right)$, an AFM transition about $150 \mathrm{~K}\left(T_{N}\right)$, and a FM transition around $100 \mathrm{~K}\left(T_{C}\right)$. Below the AFM transition temperature, interestingly, application of a high magnetic field leads to the FM and metallic state. Although the nature of the induced FM and metallic phase has not been completely understood yet, it has been suggested that the induced state might be due to the development of FM clusters in the CO and AFM matrix after the application of a magnetic field. This implies that the field-induced state has its origin in a change of the electronic state of the Mn ions and is not directly related to the Pr moments. Neutron diffraction study on $\mathrm{Pr}_{0.7} \mathrm{Ca}_{0.3} \mathrm{MnO}_{3}$ by Cox et al, however, revealed the presence of ordered Pr $4 \mathrm{f}$ moments below $60 \mathrm{~K}$ [12]. Very recently, Dho et al also reported evidence of long range ordering of the Pr spins in $\operatorname{Pr}_{0.8} \mathrm{Ca}_{0.2} \mathrm{MnO}_{3}$, implying that the field induced $\mathrm{FM}$ and metallic state might be associated with the development of the ordered Pr moments [13].

In this paper, we present various magnetization and relaxation data for two Pr-doped manganites $\operatorname{Pr}_{0.65} \mathrm{Ca}_{0.35} \mathrm{MnO}_{3}$ and $\operatorname{Pr}_{0.82} \mathrm{Na}_{0.18} \mathrm{MnO}_{3}$ with almost identical doping concentration. Our results clearly reveal that the field induced FM moment is present in both materials. Furthermore, this FM phase is remarkably sustained for $10^{5} \mathrm{~s}$ even after the field is removed. It is the main purpose of this paper to show that the field induced irreversible FM state is different from the metamagnetic transition as well as the typical spontaneous FM moment. In addition, we discuss the origin of this irreversible FM state, which might be due to the ordered Pr moments that are able to control the direction of neighboring Mn 
spins.

\section{EXPERIMENTALS}

Several single crystals of $\operatorname{Pr}_{0.65} \mathrm{Ca}_{0.35} \mathrm{MnO}_{3}$, corresponding to $35 \%$ doping of holes onto the Mn sites, were grown by a floating zone method using an infrared image furnace. Many attempts to grow single crystal of $\operatorname{Pr}_{0.82} \mathrm{Na}_{0.18} \mathrm{MnO}_{3}$ with the same floating zone method were unsuccessful mainly because the sodium is rapidly evaporated upon melting. For the Nadoped compound, ploycrystalline samples synthesized by conventional solid state reaction were thus used. X-ray powder diffraction data of the crushed crystalline and polycrystalline samples at room temperature show them to be a single phase, in which both samples adopt an orthorhombic Pnma symmetry. The magnetization data were collected on a superconducting quantum interference device (SQUID) magnetometer, and the resistivity data were collected using four-point method.

\section{RESULTS}

\section{A. Irreversible field-induced phase transition in $\operatorname{Pr}_{0.65} \mathrm{Ca}_{0.35} \mathrm{MnO}_{3}$}

The temperature-dependent dc magnetization and resistivity data of $\mathrm{Pr}_{0.65} \mathrm{Ca}_{0.35} \mathrm{MnO}_{3}$, taken under both zero-field-cooled (ZFC) and field-cooled (FC) conditions, are presented in Figure 1. The magnetization curves below $3 \mathrm{~T}$ did not reveal any discernible change indicative of a FM transition. Instead, two broad humps are seen at 160 and $230 \mathrm{~K}$, which correspond to AFM and charge ordering transitions, respectively. Above 4 T, a clear fieldinduced transition is observed near $100 \mathrm{~K}$, indicating that a metamagnetic transition from AFM to FM takes place by the application of a magnetic field. In addition, the $\mathrm{M}(T)$ curves display a large splitting between the FC and ZFC curves below $25 \mathrm{~K}$, indicating the existence of spin-glass-like state in the low temperature region. The temperature dependent resistivity data shown in the bottom panel are well correlated with the $\mathrm{M}(T)$ curves discussed above. 
The resistivity curve exhibits a steep increase near $T_{C O}$ and an insulator-to-metal (I-M) transition occurs in the presence of a high magnetic field near the field-induced FM transition temperature. A clear splitting between the ZFC and FC resistivity data is observed below $25 \mathrm{~K}$, which is consistent with the splitting in the $\mathrm{M}(T)$ data.

To further understand the nature of the field-induced FM transition, we have explored the magnetization as a function of magnetic field at selected temperatures (Figure 2). Each panel includes an initial magnetization curve and a hysteresis loop between $-5.5 \mathrm{~T}$ and +5.5 $\mathrm{T}$. The $\mathrm{M}(\mathrm{H})$ curves measured at $50 \mathrm{~K}$ exhibit a distinctive metamagnetic transition from AFM to FM near $4 \mathrm{~T}$. When the magnetic field is released, the field-induced FM state is returned to an initial AFM state near 2.5 T. As can be seen in the middle panel of Figure 2, however, the $\mathrm{M}(\mathrm{H})$ data at $20 \mathrm{~K}$ show that the initial field sweep curve yields an irreversible change. In particular, the induced FM moment partially remains even after field quenching. Furthermore, at $5 \mathrm{~K}$ the hysteresis loop exhibits little evidence of an AFM signature although the initial magnetization curve shows a metamagnetic transition. The hysteresis curve indicates a soft FM character without hysteretic energy loss. This FM state is thus apparent in the low temperature region, implying that the field-induced FM state becomes stable after the measurement of the initial magnetization curve. An important feature is that this irreversible field-induced phase transition is different from a reversible metamagnetic transition typically observed in the AFM material.

Figure 3 shows the resistivity curves for $\operatorname{Pr}_{0.65} \mathrm{Ca}_{0.35} \mathrm{MnO}_{3}$ as a function of magnetic field measured at 5 and $50 \mathrm{~K}$. At $50 \mathrm{~K}$, the resistivity suddenly drops near $4 \mathrm{~T}$, indicating that the charge-ordered insulating phase is melted into the FM metallic state by the external field. Upon reducing the magnetic field, the metallic phase is gradually changed into the charge-ordered ground state. The resistivity data between $-5.5 \mathrm{~T}$ and $5.5 \mathrm{~T}$ also show a hysteretic behavior as found in the magnetization data. However, the resistivity curve at $5 \mathrm{~K}$ shows a distinctive I-M transition about $4 \mathrm{~T}$ and the field-induced metallic state is sustained even after removing the magnetic field. All these transport behaviors are well consistent with the magnetization data discussed above. 
The stability of the field-induced FM moments in the low temperature region was examined by measuring the long-time relaxation of the dc magnetization. The sample was cooled with a field of $5 \mathrm{~T}$ down to the measured temperature $(5 \mathrm{~T})$ and the time dependent magnetization data were then collected immediately after setting the magnetic field at 0.3 T. Figure 4 shows the $\mathrm{M}(\mathrm{t})$ curves as a function of time. An important feature is that the magnetization at $5 \mathrm{~K}$ was roughly constant for $10^{5} \mathrm{~s}$ without any noticeable change. With increasing temperature, however, the magnetization gradually relaxes to the value of the AFM state. These results suggest that the field-induced FM moments are very stable at 5 K but are gradually destroyed at higher temperature.

The temperature dependence of the field-annealed FM moment was also investigated in order to compare with the ZFC magnetization curve shown in Figure 1 . The sample was cooled down to $5 \mathrm{~K}$ in $5 \mathrm{~T}$, followed by removing the magnetic field for $10^{3} \mathrm{~s}$. The magnetization data (filled symbol) were then collected immediately after setting a field of $0.3 \mathrm{~T}$. As can be seen in the $\mathrm{M}(T)$ curve given in Figure 5, a distinctive magnetic transition from FM to AFM is observed near $25 \mathrm{~K}$. The ac susceptibility data obtained in zero field also exhibits almost identical temperature dependent behavior. On the other hand, the ZFC magnetization curve (open symbol) obtained at the same magnetic field (0.3 T) does not show any tangible FM transition over the entire temperature range. The magnetic transition from FM to AFM near $25 \mathrm{~K}$ is also accompanied by a metal-insulator transition. This clearly suggests that the FM transition below $25 \mathrm{~K}$ results from the field annealing.

\section{B. Irreversible field-induced phase transition in $\operatorname{Pr}_{0.82} \mathrm{Na}_{0.18} \mathrm{MnO}_{3}$}

In order to probe the role of the Pr concentration, we have also investigated the magnetization and transport properties of $\mathrm{Pr}_{0.82} \mathrm{Na}_{0.18} \mathrm{MnO}_{3}$. Since the Na-doped compound has almost the same doping level as well as the same average A-site ionic size by comparison with those of $\operatorname{Pr}_{0.65} \mathrm{Ca}_{0.35} \mathrm{MnO}_{3}$, its charge ordering and $\mathrm{AFM}$ transitions occur near the temperatures observed in $\operatorname{Pr}_{0.65} \mathrm{Ca}_{0.35} \mathrm{MnO}_{3}[3,10]$. An important difference, however, 
is that the $\mathrm{Pr}$ concentration is much larger in $\mathrm{Pr}_{0.82} \mathrm{Na}_{0.18} \mathrm{MnO}_{3}$ than in $\mathrm{Pr}_{0.65} \mathrm{Ca}_{0.35} \mathrm{MnO}_{3}$. This would enable to see the pronounced Pr effect in the Na-doped sample that is crucial to elucidate the origin of the irreversible field induced ferromagnetism.

Figure 6 displays the magnetization curves of $\operatorname{Pr}_{0.82} \mathrm{Na}_{0.18} \mathrm{MnO}_{3}$ as a function of magnetic field at selected temperatures. The hysteresis loops between $+5.5 \mathrm{~T}$ and $-5.5 \mathrm{~T}$ (filled circle) were recorded just after the initial measurement from $0 \mathrm{~T}$ to $+5.5 \mathrm{~T}$ (open circle). Above $100 \mathrm{~K}$, the $\mathrm{M}(\mathrm{H})$ curves exhibit a metamagnetic transition from AFM to FM with increasing the magnetic field. The critical magnetic field decreases with decreasing the temperature. In addition, the field-induced FM state is returned to an initial AFM state upon removal of the magnetic field. At $80 \mathrm{~K}$, however, the $\mathrm{M}(\mathrm{H})$ data show that the initial field sweep curve yields an irreversible change similar to that of $\mathrm{Pr}_{0.65} \mathrm{Ca}_{0.35} \mathrm{MnO}_{3}$ measured at $20 \mathrm{~K}$. In particular, the induced FM moment is sustained even after field quenching. When the magnetic field is swept from $0 \mathrm{~T}$ to $-5.5 \mathrm{~T}$, the $\mathrm{M}(\mathrm{H})$ curve displays both FM and AFM signatures at low and high field regions, respectively. Below $60 \mathrm{~K}$, the initial magnetization curve shows a metamagnetic transition but other $\mathrm{M}(\mathrm{H})$ data clearly exhibit a typical soft FM feature. It is worthwhile to note that the field-induced FM state is found in much broader region in $\operatorname{Pr}_{0.82} \mathrm{Na}_{0.18} \mathrm{MnO}_{3}$ compared with that of $\mathrm{Pr}_{0.65} \mathrm{Ca}_{0.35} \mathrm{MnO}_{3}$, which is due to the large Pr concentration in the Na-doped compound.

The temperature dependence of the field-annealed FM moment of $\mathrm{Pr}_{0.82} \mathrm{Na}_{0.18} \mathrm{MnO}_{3}$ was also investigated, which is given in Figure 7. The sample was cooled down to $5 \mathrm{~K}$ in the presence of a magnetic field of $5 \mathrm{~T}$, followed by quenching the field for $10^{3} \mathrm{~s}$. The temperature dependent magnetization data (filled squares) were then collected immediately after setting a field of $0.3 \mathrm{~T}$. As can be seen in the $\mathrm{M}(\mathrm{T})$ curve, a distinctive FM transition is observed near $90 \mathrm{~K}$. On the other hand, the magnetization data without the field annealing (open squares) show little indication of a FM transition. These magnetic data also coincide with the resistivity curves. The field-annealed sample shows a distinctive I-M transition near the FM transition temperature while the sample without the field annealing is insulating. An interesting feature to be noted is that the irreversible field-induced FM phase is also 
discovered in other Pr-doped manganites such as $\mathrm{Pr}_{0.7} \mathrm{Ca}_{0.3} \mathrm{MnO}_{3}, \mathrm{Pr}_{0.67} \mathrm{Ca}_{0.33} \mathrm{MnO}_{3}$, and $\operatorname{Pr}_{0.65} \mathrm{Ca}_{0.35} \mathrm{MnO}_{3}$ [14]. The $T_{C}$ of the field-induced FM phase monotonously decreases from $60 \mathrm{~K}$ to $25 \mathrm{~K}$ with decreasing the Pr concentration. More interestingly, no irreversible field-induced FM transition behavior is found in $\operatorname{Pr}_{0.6} \mathrm{Ca}_{0.4} \mathrm{MnO}_{3}$ with the low $\operatorname{Pr}$ content, clearly suggesting that the field-induced FM exchange coupling is dependent on the $\operatorname{Pr}$ concentration.

\section{DISCUSSIONS}

It is worthwhile to address that the FM moment of Pr-doped manganites, produced by field annealing is different from a spontaneous magnetic moment found in a typical FM material as well as in the reversible metamagnetic transition. A distinctive feature in the field-annealed FM phase is that the ferromagnetism is induced by field annealing and survives even after field quenching. Now the question is how this irreversible field-induced FM phase in $\operatorname{Pr}_{0.65} \mathrm{Ca}_{0.35} \mathrm{MnO}_{3}$ and $\operatorname{Pr}_{0.82} \mathrm{Na}_{0.18} \mathrm{MnO}_{3}$ is stabilized in the $\mathrm{CO}$ and AFM matrix. To understand this unusual phenomenon, we employ a schematic energy diagram (Figure 8) that includes two local minima corresponding to the AFM and FM states. In zero magnetic

field, the ground state of the two compounds is the pseudo CE-type CO and AFM state. As given in Figure 8(a), the FM state is not stable at this stage. In the presence of a high magnetic field, however, the FM phase is stabilized by means of the Zeemann energy term $(-\mathrm{M} \cdot \mathrm{H})$, which is depicted in Figure 8(b). In the ordinary AFM material, the field-induced FM state immediately goes back to the AFM ground state when the field is removed. The field-induced FM phase in the Pr-doped manganites to be discussed is irreversible and stable even after quenching the field. This can be described by the introduction of pinning potential barrier (U), given in Figure 8(c). The only way to destabilize the FM state is the increase of the thermal fluctuation in the high temperature region. Above a critical temperature $\left(T_{0}\right)$, the thermal fluctuation abruptly induces a transition from FM to AFM, as seen in Figure 5 and 7. Accordingly, we can simply estimate that the pinning potential barrier is $\sim k_{B} T_{0}$, 
where $k_{B}$ is the Boltzmann constant, which is corresponding to about 2-8 meV in Pr-doped manganite samples. Our simple energy diagram well explains the reversible and irreversible FM states and also agrees with the $\mathrm{M}(\mathrm{H})$ curves given in Figure 2 and 6.

Another important issue to be addressed is that spin reorientation is the most likely cause of the irreversible field-induced FM state found in the Pr-doped manganites [15-18]. In zero magnetic field, FM exchange coupling between the Pr $4 \mathrm{f}$ and Mn 3d spins is negligible because the Pr spins have no net moments in the AFM matrix. With an increase in the magnetic field, however, the Mn spins undergo a metamagnetic transition and simultaneously the localized Pr spins change to an ordered state. Consequently, the FM exchange coupling between the Pr and Mn spins is operative, making the field-induced FM state of Mn spins stable. The origin of this unique FM state thus appears to be associated with the coupled interaction between trivalent $\operatorname{Pr}$ ions and mixed valent Mn ions. Probably the ordered $4 \mathrm{f}$ Pr spin might pin the itinerant Mn spins. Namely they control the spin direction of $\mathrm{Mn}$ 3d electrons through the FM exchange coupling, which eventually leads to the development of the ferromagnetism. Accordingly, this field annealed FM state is pinned by the ordered 4f spins, which makes the FM phase stable. It is conceivable that the Pr $4 \mathrm{f}$ electrons may act as a pinning center for pinning itinerant charge carriers. This anomalous irreversible switching of the FM interaction is strongly dependent upon the order-disorder transition of the localized Pr spins.

Finally, it is worthy to mention that this field-induced irreversible FM phase is not easily observed in the $\mathrm{Nd}$-doped samples $\mathrm{Nd}_{1-x} \mathrm{Ca}_{x} \mathrm{MnO}_{3}$ although sizes and magnetic moments of the $\operatorname{Pr}$ and Nd ions are similar [19,20]. One likely cause of this difference is ascribed to the different electronic states between the $\mathrm{Pr}$ and $\mathrm{Nd}$ ions. Unlike the $\mathrm{Nd}$ ion that has predominantly a $3+$ state, the $\operatorname{Pr}$ ion can have a $4+$ state as well as a $3+$ state. This mixed valent nature appears to induce the strong FM exchange coupling in the Pr-doped manganite efficiently. Another plausible cause is due to subtle size difference between the Nd and Pr ions. The smaller Nd ion leads to slightly larger lattice distortion, which makes the FM exchange coupling between the Nd $4 \mathrm{f}$ and Mn 3d spins coupling difficult. 


\section{CONCLUSIONS}

We have discovered a new type of ferromagnetism in Pr-doped manganites. The observed ferromagnetism is quite unusual in that the field annealing yields an irreversible field-induced FM state. This irreversible field-induced FM state is due to an irreversible switching of the FM interaction between the Pr and Mn spins by the magnetic field. If one calls a spontaneous magnetic moment typically observed in a FM material type I ferromagnetism, then this field-induced ferromagnetism might be dubbed type II ferromagnetism. We have adopted this terminology from type II superconductor. This is because although they are intrinsically different, they have some similar features, particularly in a mixed state. The FM moment induced by the ordered Pr spins that pin the itinerant charge carriers will play an important role in determining the physical properties of $\mathrm{Pr}_{0.65} \mathrm{Ca}_{0.35} \mathrm{MnO}_{3}$ and $\operatorname{Pr}_{0.82} \mathrm{Na}_{0.18} \mathrm{MnO}_{3}$. Indeed, we have found that the order-disorder transition of localized $\mathrm{Pr}$

spins is strongly correlated with a metal-insulator transition in the field-annealed sample of $\operatorname{Pr}_{0.65} \mathrm{Ca}_{0.35} \mathrm{MnO}_{3}$.

\section{ACKNOWLEDGEMENT}

The Creative Research Initiative Program supported this work. 


\section{REFERENCES}

* Author to whom correspondce should be addressed : nhhur@kriss.re.kr

[1] C. Zener, Phys. Rev. 82, 403 (1951).

[2] Y. Tokura, Colossal magnetoresistance oxides (Gordon and Breach Science Publishers, edited by Tokura, Y., 2000).

[3] J. M. D. Coey and M. Viret, Adv. Phys. 48, 167 (1999).

[4] H. Yoshizawa, H. Kawano, Y. Tomioka, and Y. Tokura, Phys. Rev. B 52, R13145 (1995); J. Phys. Soc. Japan 65, 1043 (1996).

[5] Y. Tomioka, A. Asamitsu, H. Kuwahara, Y. Moritomo, and Y. Tokura, Phys. Rev. B 53, R1689 (1996).

[6] Y. Moritomo, H. Kuwahara, Y. Tomioka, and Y. Tokura, Phys. Rev. B 55, 7549 (1997).

[7] P. G. Radaelli, R. M. Ibberson, D. N. Argyriou, H. Casalta, K. H. Andersen, S.-W. Cheong, and J. F. Mitchell, Phys. Rev. B 63, 172419 (2001).

[8] I. G. Deac, J. F. Mitchell, P. Schiffer, Phys. Rev. B 63, 172408 (2001).

[9] Z. Jirák, S. Krupička, Z. Šimša, M. Dlouhá, and S. Vratislav, J. Magn. Magn. Mater. 53, 153(1995).

[10] J. Hejtmánek, Z. Jirák, J. Šebek, A. Strejc, and M. Hervieu, J. App. Phys. 89, 7413 (2001).

[11] T. Satoh, Y. Kikuchi, K. Miyano, E. Pollert, J. Hejtmanek, and Z. Jirak, Phys. Rev. B 65, $125103(2002)$.

[12] D. E. Cox, P. G. Radaelli, M. Marezio, and S.-W. Cheong, Phys. Rev. B 57, 3305 (1998).

[13] J. Dho, W. S. Kim, N. H. Hur , K. W. Lee, H. S. Oh, and Y. N. Choi, Solid State 
Comm. 123, 441 (2002).

[14] J. Dho, W. S. Kim, E. O. Chi, N. H. Hur, S. H. Park, and H. -C. Ri, Solid State Comm. 125, 143 (2003).

[15] S. V. Fridrikh et al. , Phys. Rev. Lett. 79, 4661 (1997)

[16] M. D. Lumsden, B. C. Sales, D. Mandrus, S. E. Nagler, and J. R. Thompson, Phys. Rev. Lett. 86, 159 (2001).

[17] N. Tsujii, H. Kitazawa, H. Suzuki, M. Imai, and G. Kido, Journal of Phys. Soc. Japan 71, 1852 (2002).

[18] M. R. Lees, J. Barratt, G. Balakrishnan, D. M. Paul, and M. Yethiraj, Phys. Rev. B 52, R14303 (1995).

[19] M. Tokunaga, N. Miura, Y. Tomioka, and Y. Tokura, Phys. Rev. B 57, 5259 (1998).

[20] X.-J. Fan, H. Koinuma, T. Hasegawa, Phys. Rev. B 65, 144401 (2002). 


\section{FIGURES}

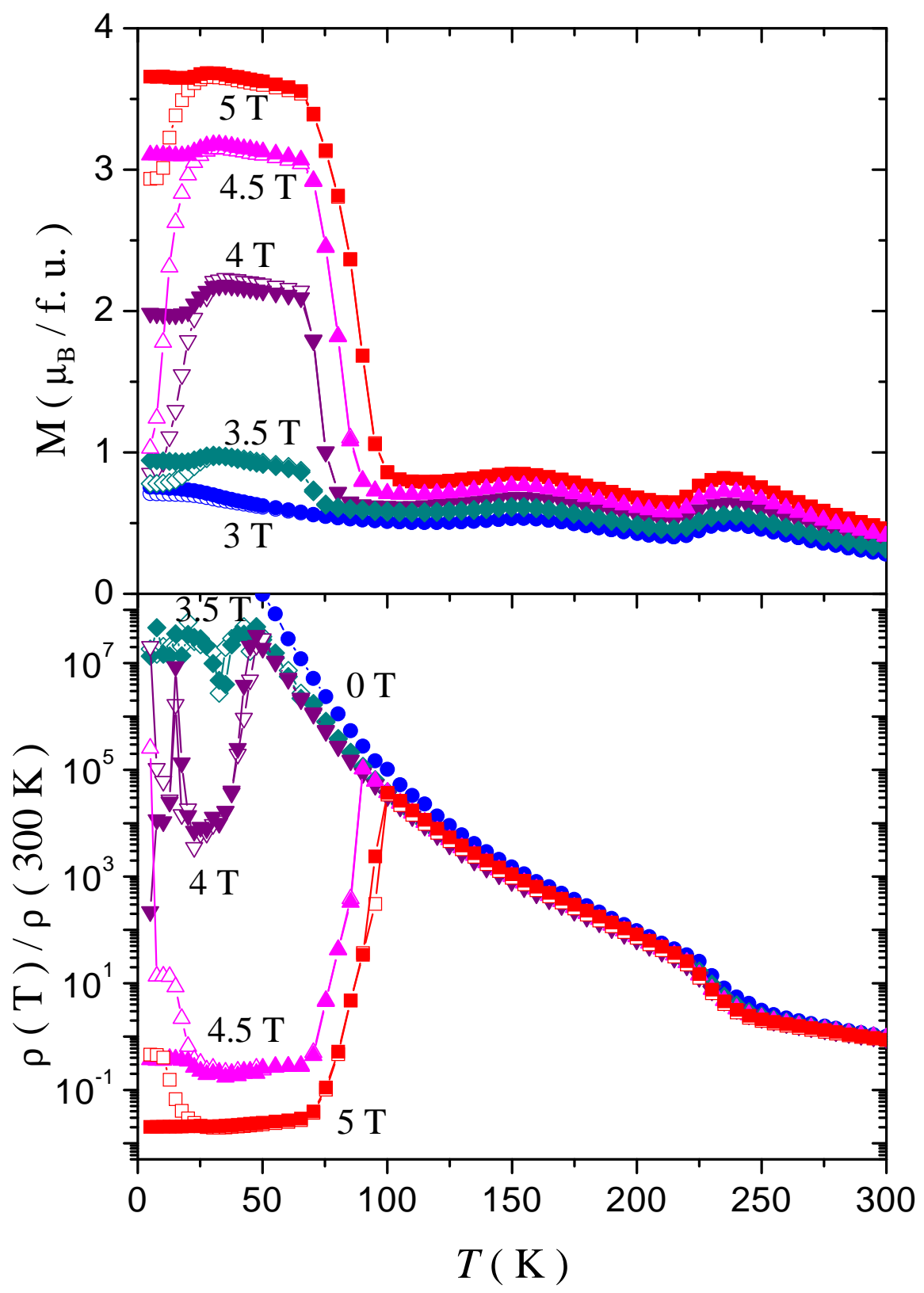

FIG. 1. Temperature dependent magnetization of $\operatorname{Pr}_{0.65} \mathrm{Ca}_{0.35} \mathrm{MnO}_{3}$ taken in various magnetic fields during both the zero-field-cooled (ZFC, open symbol) and field-cooled (FC, filled symbol) runs. 


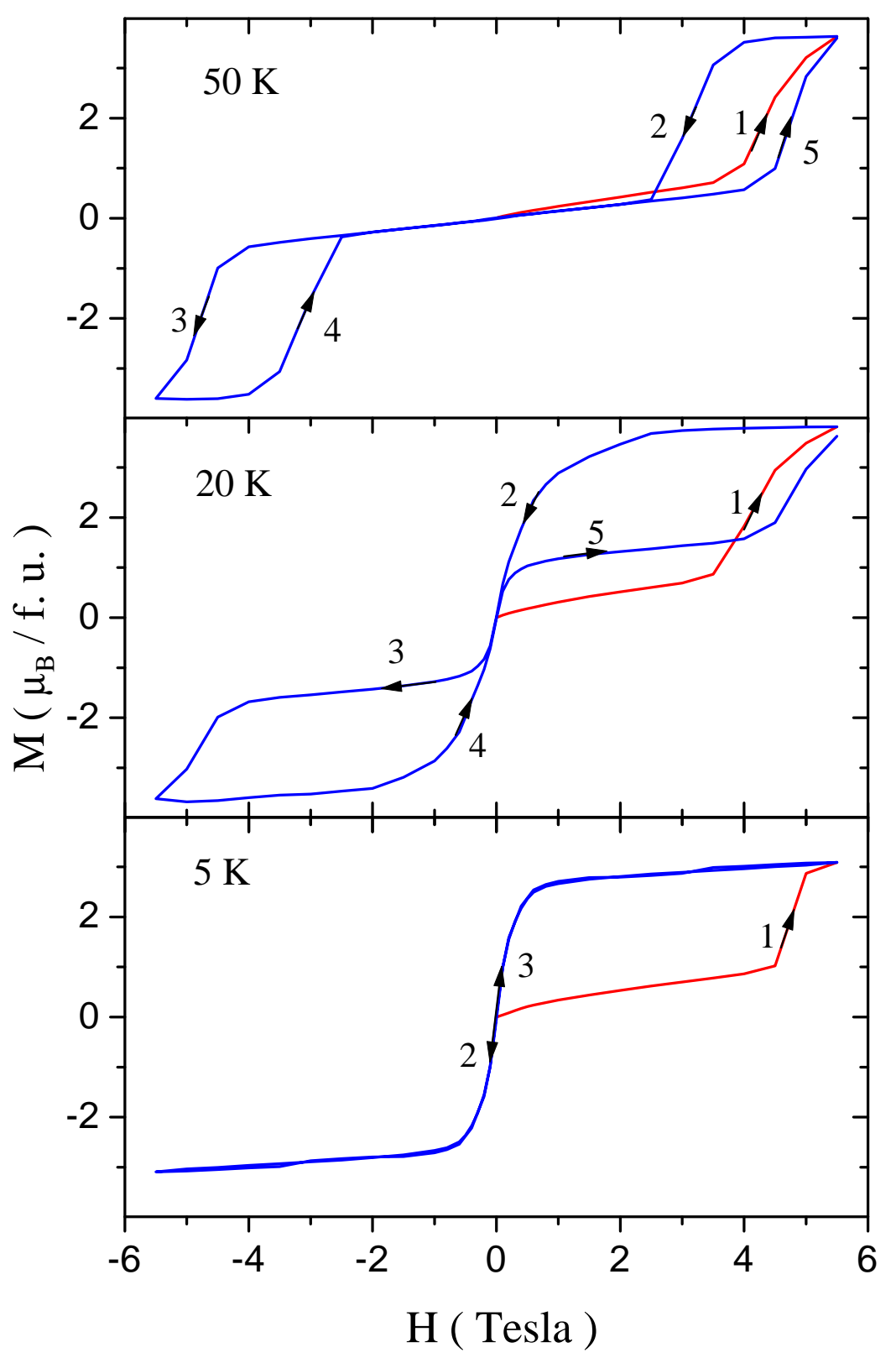

FIG. 2. Field dependence of magnetization measured at selected temperatures for $\operatorname{Pr}_{0.65} \mathrm{Ca}_{0.35} \mathrm{MnO}_{3}$. Measurements have been carried out in numerical sequence. 


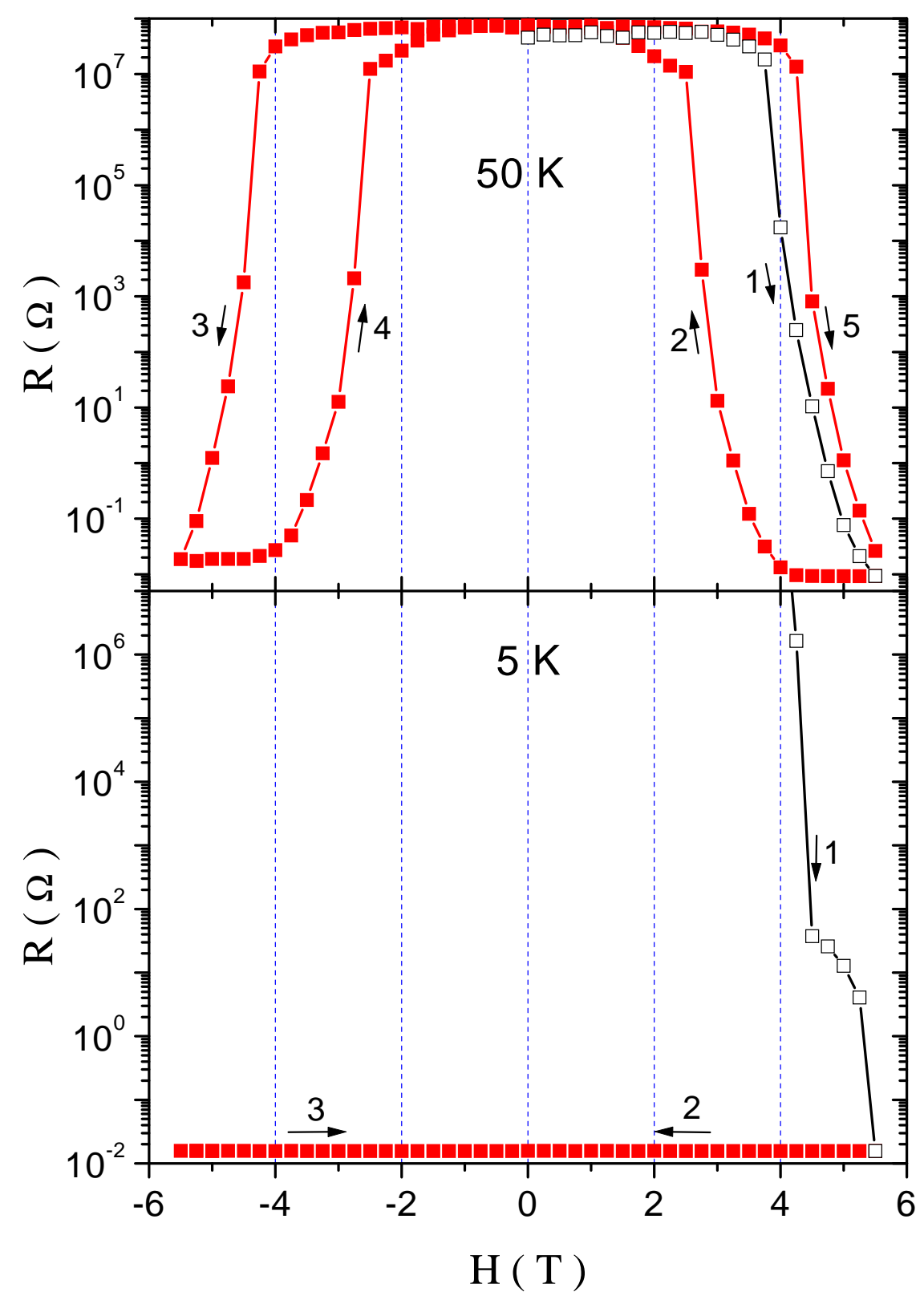

FIG. 3. Field dependence of resistivity measured at selected temperatures for $\operatorname{Pr}_{0.65} \mathrm{Ca}_{0.35} \mathrm{MnO}_{3}$. Measurements have been carried out in numerical sequence. 


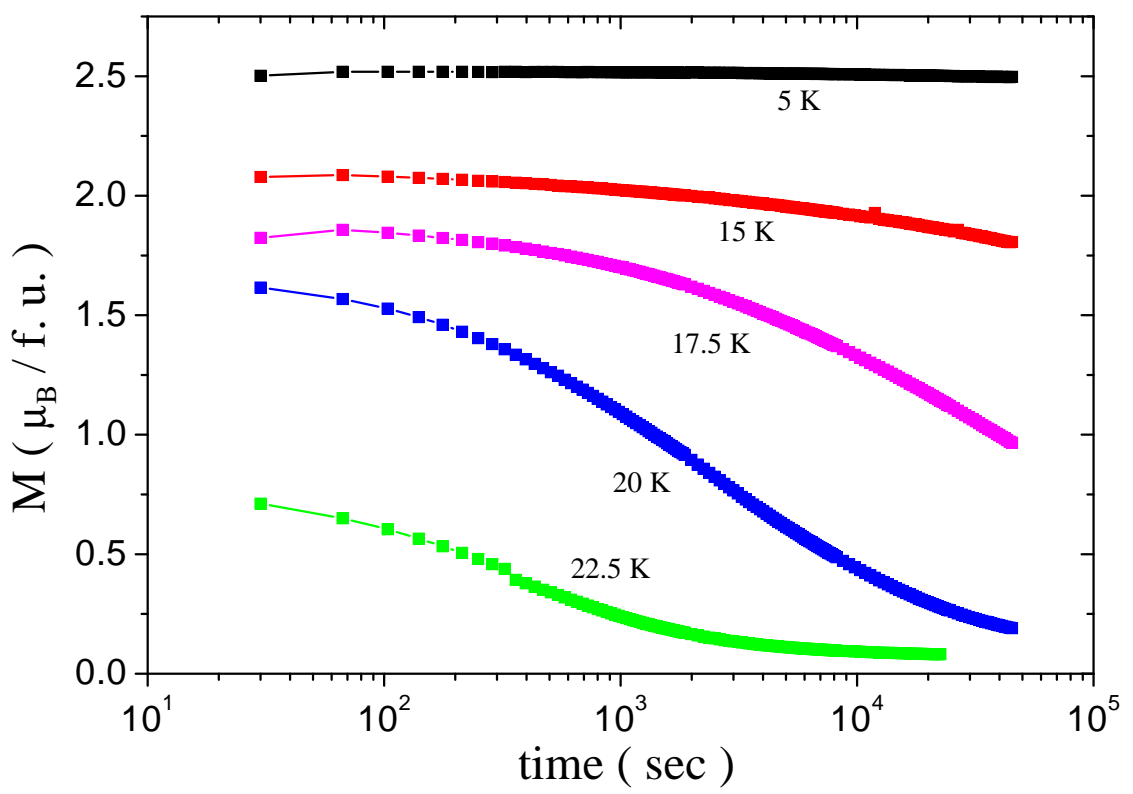

FIG. 4. Time dependence of magnetization for $\operatorname{Pr}_{0.65} \mathrm{Ca}_{0.35} \mathrm{MnO}_{3}$ at several temperatures. The sample was initially cooled from $300 \mathrm{~K}$ down to the measured temperature under $5 \mathrm{~T}$, followed by measuring the time dependent magnetization immediately after setting the field to $0.3 \mathrm{~T}$. 


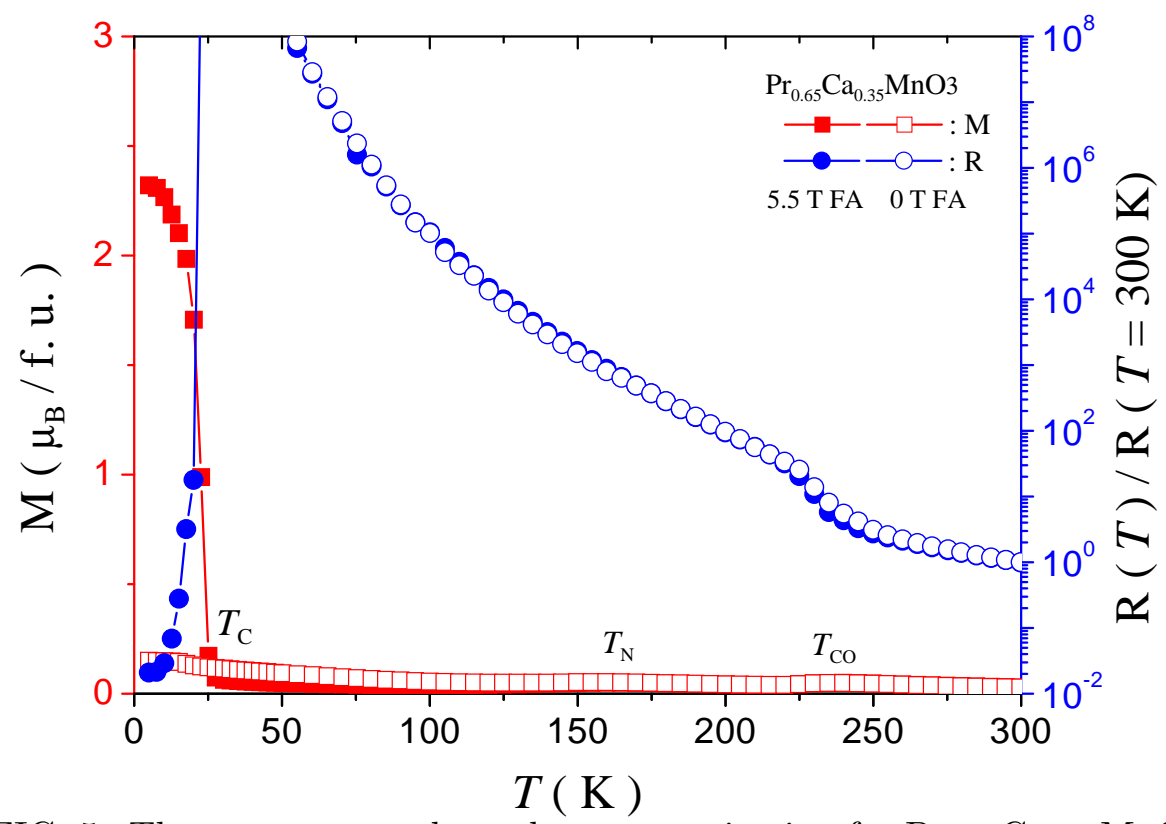

FIG. 5. The temperature dependent magnetization for $\operatorname{Pr}_{0.65} \mathrm{Ca}_{0.35} \mathrm{MnO}_{3}$ measured after magnetic field-annealing (FA). The sample was cooled down to $5 \mathrm{~K}$ at $0 \mathrm{~T}$ (open symbol) and $5 \mathrm{~T}$ (filled symbol) in respective runs, followed by removing the magnetic field for $10^{3} \mathrm{~s}$. The magnetization and resistivity measurements were performed in $0.3 \mathrm{~T}$ and $0 \mathrm{~T}$, respectively. All the data were collected in warming cycle. 

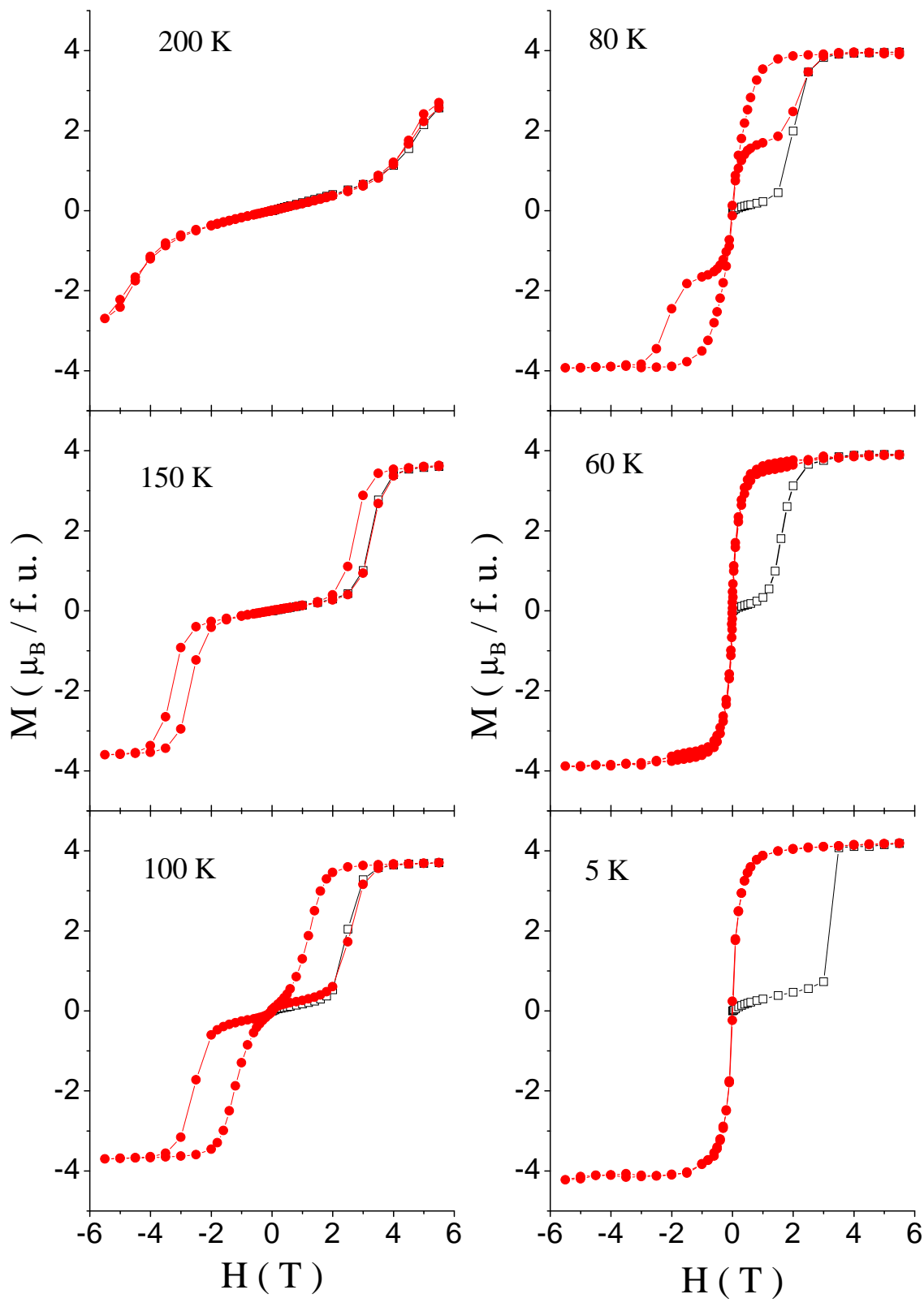

FIG. 6. The field dependent magnetization curves measured at selected temperatures for $\operatorname{Pr}_{0.82} \mathrm{Na}_{0.18} \mathrm{MnO}_{3}$. The open squares represent the initial magnetization data and the filled squares denote the subsequent hysteresis loop. 


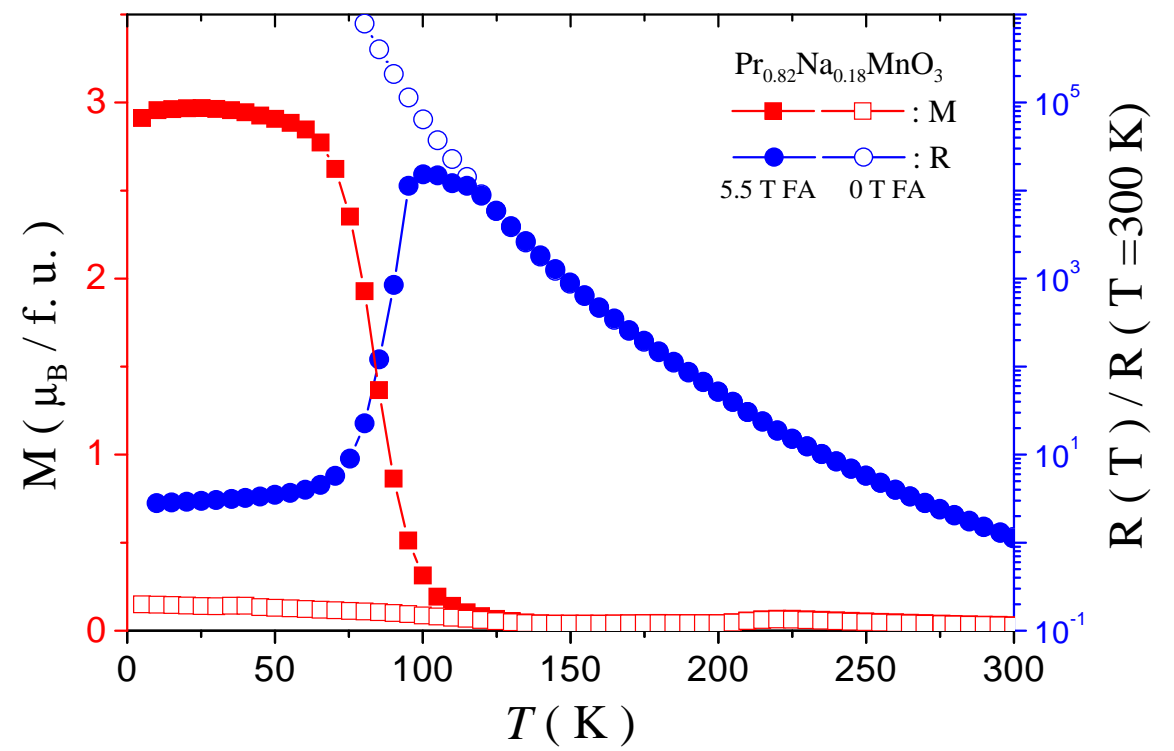

FIG. 7. The magnetization and resistivity curves for $\operatorname{Pr}_{0.82} \mathrm{Na}_{0.18} \mathrm{MnO}_{3}$ as a function of temperature. The sample was cooled down to $5 \mathrm{~K}$ at $0 \mathrm{~T}$ (open symbols) and $5 \mathrm{~T}$ (filled symbols), followed by removing the magnetic field for $10^{3} \mathrm{~s}$. The magnetization and resistivity measurements were performed in $0.3 \mathrm{~T}$ and $0 \mathrm{~T}$, respectively. All the data were collected in the warming cycles. 
(a) $\mathbf{H}=\mathbf{0} \mathrm{T}$

(b) high magnetic field

(d) $\mathbf{H}=\mathbf{0} \mathbf{T}$ irreversible

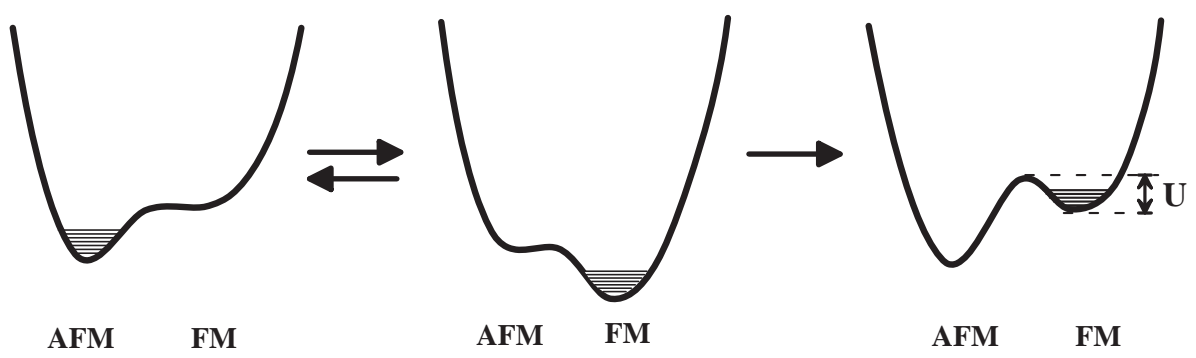

FIG. 8. Schematic energy diagrams of FM and AFM states with and without the external magnetic field, in which two local minima represent potential energy states of AFM and FM phases. 